\title{
Rapid Distension of Abdomen in a Young Woman
}

\author{
Gonzalez L*
}

Acute Physician in AAU, Great Western Hospital, Swindon, United Kingdom

*Corresponding author: Gonzalez L, Acute Physician in AAU, Great Western Hospital, Swindon, United Kingdom, E-mail: lauravgonzalez@googlemail.com

Citation: Gonzalez L (2017) Rapid Distension of Abdomen in a Young Woman. SAJ Case Reports 4: 404

Article history: Received: 23 October 2017, Accepted: 28 November 2017, Published: 30 November 2017

\section{Introduction}

A 35 years old lady was admitted to the medical assessment Unit with 2 weeks of progressive breathlessness and distended abdomen. On examination she did have a large right pleural effusion and very large distended abdomen.

Blood test including FBC, U\&E, LFT and calcium were normal with a CRP of 33. She underwent a CT abdomen which demonstrated a very large mass in the abdomen, multicystic, suggestive of ovarian origin (Figure 1). The uterus was bulky, and there was a large right pleural effusion.
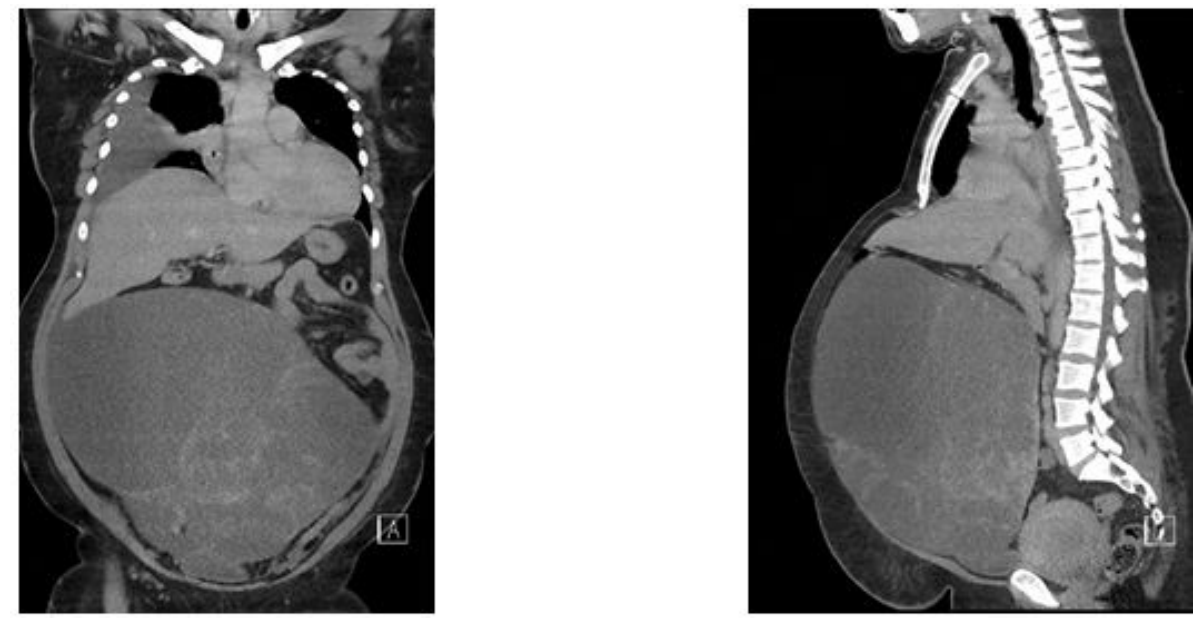

Figure 1: CT scan of abdomen: large multicystic mass in abdomen and right pleural effusion

The pleural effusion was drained and under gynaecologist she did have surgery: total hysterectomy, bilateral salpingo-oopherectomy, omentectomy and peritoneal washing for a large $40-50 \mathrm{~cm}$ multiloculated tumour.

Histology demonstrated a stage 1c grade 2 endometrioid ovarian adenocarcinoma and stage 1a endometrial carcinoma. Oncologist advised to add adjuvant quemotherapy to her treatment with Carboplatin and Paclitaxel. She moved to Fiji after her relative's death and not follow up has been possible.

Ovarian cysts can vary in size, from $<1 \mathrm{~cm}$ to $>10 \mathrm{~cm}$. A large cyst does not predict if cancer is present.

Endometrioid ovarian cancer represents a 10-15\% of primary ovarian tumors. 15\% coexists with endometriosis. $15-30 \%$ of patients will have synchronous endometrial hyperplasia or carcinoma. This 2 types of cancer share many histological and molecular alterations, likely due to a common endometrial epithelial precursor cell of origin [1].

Endometriosis is thought to occur via retrograde menstruation, and the endometrial cells settle as an endometriotic cyst within the ovary. The menstruation like blood and necrotic tissue is trapped in the cyst causing oxidative stress and hypoxic environment leading to DNA damage and mutation accumulation. 
Women with endometrioid cancer present at a younger age and with earlier stage disease than those with serous ovarian cancer $[2,3]$. The endometroid histology is associated with better survival rates than in serous adenocarcinoma of the ovary, and is correlated with earlier presentation. Patients with concurrent endometrioid ovarian and endometrial malignancies had a survival advantage compared with those with ovarian malignancies alone [2].

\section{References}

1. McConechy MK, Ding J, Senz J, Yang W, Melnyk N, et al. (2014) Ovarian and endometrial endometrioid carcinomas have distinct CTNNB1 and PTEN mutation profiles. Mod Pathol 27: 128-34.

2. Storey DJ, Rush R, Stewart M, Rye T, Al-Nafussi, et al. (2008) A Endometrioid epithelial ovarian cancer: 20 years of prospectively collected data from a single center. Cancer 112: 2211-20.

3. Bruno DM, Alexandra S, Endometrioid carcinoma of the ovary. rID: 13420. 